\title{
The Effect of Oral Probiotic Streptococcus Salivarius K12 on Candida Albicans Biofilm Formation
}

Munirah Mokhtar ${ }^{1}$, Alia Risma Rismayuddin², Ridhwan Abdul Wahab¹, Muhamad Ashraf Rostam ${ }^{1}$, Mohd Hamzah Mohd Nasir ${ }^{3}$, Mohd Hafiz Arzmi

${ }^{1}$ Department of Biomedical Sciences, Kuliyyah of Allied Health Sciences, International Islamic University Malaysia

2Department of Basic Medical Sciences, Kulliyyah of Nursing, International Islamic University Malaysia

3Department of Biotechnology, Kulliyyah of Science, International Islamic University Malaysia

${ }^{4}$ Department of Fundamental Dental and Medical Sciences, Kulliyyah of Dentistry, International Islamic University Malaysia.

Introduction: Oral cancer is the sixth most common cancer worldwide with Candida albicans infection being one of the aetiological factors for the disease. Meanwhile, Streptococcus salivarius $\mathrm{K} 12$ is an oral probiotic that is beneficial to the oral cavity. The objective of the present study is to determine the effect of S. salivarius K12 on $\mathrm{C}$. albicans biofilm-forming ability with the hypothesis that $\mathrm{S}$. salivarius $\mathrm{K} 12$ inhibits biofilm formation of $C$. albicans Materials and method: To assess the effect of S. salivarius $\mathrm{K} 12$ on $\mathrm{C}$. albicans biofilm formation, S. salivarius K12, lab strain C. albicans MYA-4901 and clinical isolates from oral cancer, ALC2 and ALC3 were grown in both nutrient broth (NB) and RPMI. In a mono-species biofilm, $10^{5}$ of $\mathrm{C}$. albicans cells and $10^{6}$ of $\mathrm{S}$. salivarius $\mathrm{K} 12$ cells were grown separately in a 96-well plate. In contrast, both microorganisms were combined for polymicrobial biofilms with similar cell numbers as in mono-species. The biofilms were incubated for 72 hours at $37^{\circ} \mathrm{C}$ and the media were replenished every 24 hours. Finally, the crystal violet assay was conducted, and the optical density was measured at OD620nm. Results: Polymicrobial biofilms of C. albicans (MYA-4901 and ALC3) with S. salivarius $\mathrm{K} 12$ when grown in NB, exhibited a decrease by $64.5 \pm 25.8 \%$ and $83.7 \pm 5.4 \%$, respectively when compared to the expected biofilms which were predominated by yeast form. Furthermore, polymicrobial biofilms of C. albicans (ALC2 and ALC3) with S. salivarius $\mathrm{K} 12$ showed a decrease by $62.5 \pm 25.6 \%$ and 55.9 $\pm 17.1 \%$, respectively when compared to the expected biofilms when grown in RPMI that were predominated by hyphal form. Conclusion: S. salivarius K12 inhibited polymicrobial biofilms formation of C. albicans yeast and hyphal forms, thus supported the hypothesis that $\mathrm{S}$. salivarius $\mathrm{K} 12$ inhibits biofilm formation of $\mathrm{C}$. albicans. 